Original Article

\title{
DESIGN AND EVALUATION OF BUCCAL PATCHES CONTAINING COMBINATION OF HYDROCHLOROTHIAZIDE AND ATENOLOL
}

\author{
ASHUTOSH RODA ${ }^{a}$, PRABHAKARA PRABHU ${ }^{\mathrm{b}}$, AKHILESH DUBEY ${ }^{*} \mathrm{c}$
}

aFaculty of Pharmacy, Bhagwant University, Ajmer, Rajasthan, India, bDepartment of Pharmaceutics, Shree Devi College of Pharmacy, Airport Road, Mangalore 574142, Karnataka, India, ${ }^{*}$ Department of Pharmaceutics, NGSM Institute of Pharmaceutical Sciences, Nitte University, Paneer, Deralakatte,Mangalore 575018, Karnataka, India

Email: akhilesh_intas@rediffmail.com

Received: 27 Dec 2017, Revised and Accepted: 13 Feb 2018

\begin{abstract}
Objective: Buccal patch is a non-dissolving thin matrix modified release dosage form which was developed to administer into the unconscious and less co-operative patients.

Methods: The mucoadhesive buccal patches of hydrochlorothiazide (HCZ) and atenolol (ATN) were prepared by solvent casting technique using various concentrations of sodium alginate, hydroxyl propyl methyl cellulose, carbopol 934P and sodium carboxy methyl cellulose polymer and polyvinyl alcohol as a backing layer. The formulated patches were evaluated for their physicochemical parameters like thickness, weight variation, surface $\mathrm{pH}$, content uniformity, folding endurance, swelling percentage studies and tensile strength, in vitro and ex vivo drug permeation.

Results: The infra-red (IR) spectra showed no interaction between drug and polymer. Physicochemical characteristics of all the samples were found to be satisfactory and well within the range. Swelling of the films were increased with the increasing content of the polymers and it was found that swelling front erosion was comparably slower in the formulations with the carbopol 934 and HPMC. This is probably due to their marked viscous properties and therefore formulation provided sustained release of the drug. The percentage drug content of all the formulations were found to be in the range of 97-99\%. Among the patches, FC (Carbopol 934 and HPMC) patches were considered satisfactory for maintaining the in vitro residence in the oral cavity for almost 8h. Formulations FD (with $\mathrm{CP}$ and NaCMC) and FC showed high tensile strength and \% E/B which is an indication of the strength and elasticity of the patch. The films were exhibited sustained release for more than $6 \mathrm{~h}$ which was confirmed by the in vitro release data and kinetic data reveals the combination of diffusion and erosion mechanism. The best mucoadhesive performance and matrix controlled release was exhibited by the formulation FC.
\end{abstract}

Conclusion: The formulation of HCZ and ATN mucoadhesive buccal patch was found to be satisfactory and reasonable.

Keywords: Buccal mucosa, Solvent casting method, HPMC, Hydrochlorothiazide, Atenolol, Mucoadhesive buccal patches

(C) 2018 The Authors. Published by Innovare Academic Sciences Pvt Ltd. This is an open access article under the CC BY license (http://creativecommons.org/licenses/by/4.0/) DOI: http://dx.doi.org/10.22159/ijap.2018v10i2.24460

\section{INTRODUCTION}

Mucoadhesive polymers are synthetic or natural macromolecules which are capable of attaching to mucosal surfaces. Mucoadhesive drug delivery system has been accepted as a promising strategy to prolong the residence time and to improve the specific localization of drug on various membranes. Amongst the various routes of drug delivery, the oral route is perhaps the most preferred to the patient and the clinician alike [1]. However, peroral administration of drugs has disadvantages such as hepatic first pass metabolism and enzymatic degradation within the GI tract, that prohibit oral administration of certain classes of drugs especially peptides and proteins. Consequently, other absorptive mucosae are considered as potential sites for drug administration. Transmucosal routes of drug delivery (i.e., the mucosal linings of the nasal, rectal, vaginal, ocular, and oral cavity) offer distinct advantages over per oral administration for systemic drug delivery. These advantages include a possible bypass of first pass effect, avoidance of pre-systemic elimination within the GI tract, and, also provides a better enzymatic flora for drug absorption. Most of the antihypertensive formulations are available in the market are in the form of tablets. Most of them do present varying degree of disadvantages in terms of efficacy, absorption and bioavailability and sometimes show undesirable side effects due to fluctuating plasma drug level $[2,3]$.

The effort was made here to formulate the combination of HCZ and ATN as a buccal patch to provide sustained release of drug and to bypass hepatic first-pass metabolism during the treatment of hypertension. HCZ has a half-life of 5-6 h, bioavailability is around $70 \%$ and classified as BCS class 4 systems. It is mainly eliminated by the kidney and its protein binding is approximately $70 \%$. On the other hand, ATN has a half-life of 6-7 h. Duration of action is found to be $24 \mathrm{~h}$ (once daily dosing). Absorption of the drug is found to be absorbed slowly and incompletely from GI tract (oral) and peak plasma concentration is achieved after $7 \mathrm{~h}$. The bioavailability of ATN is around $25 \%$. The combination of ATN and HCZ is available in the market as tablets, for the treatment of hypertension.

Hence, buccal patches containing the combination of HCZ and ATN was designed and evaluated.

\section{MATERIALS AND METHODS}

\section{Materials}

Hydrochlorothiazide and Atenolol were procured by Yarrow Chem. Pvt. Ltd. polyvinyl alcohol, polyvinylpyrrolidone, ethyl cellulose, hydroxy propyl methyl cellulose, sodium alginate and carbopol 934 were procured by CDH laboratory Reagent, New Delhi. All other chemicals and reagents were of analytical grade.

Methods

Preformulation study

\section{FTIR studies}

Compatibility of HCZ and ATN with the excipients was confirmed by FTIR studies. The pure drugs, HCZ and ATN with the polymers in the ratio of $1: 1$ were taken and the potassium bromide disc (pellet) method was employed to conduct the study. After collecting an interferogram of a sample containing pure HCZ and ATN and drugs with the polymers, FTIR spectroscopic analysis was performed on the interferogram to obtain the spectrum.

\section{Method of preparation}

A series of buccal patches composed of different proportions and combinations of sodium alginate (SA) $(100$ to $300 \mathrm{mg})$, hydroxypropyl methyl cellulose (HPMC) (200 to $400 \mathrm{mg}$ ), carbopol 
(CP) $934 \mathrm{P}$ (100 to $300 \mathrm{mg}$ ), and sodium carboxy methyl cellulose (NaCMC) (200 to $400 \mathrm{mg}$ ) containing ATN (200 mg) and HCZ (200 $\mathrm{mg}$ ) were prepared using a $64 \mathrm{~cm}^{2}$ petri dish by solvent casting technique. Glycerine was incorporated as a plasticizer at a concentration of $15 \% \mathrm{w} / \mathrm{w}$ of dry weight of polymers. Backing membrane was casted by pouring $4 \% \mathrm{w} / \mathrm{v}$ aqueous solution of PVA on aluminium foil in petri dishes at $42^{\circ} \mathrm{C}$ and left for $10 \mathrm{~h}$. Phosphate buffer saline, $\mathrm{pH} 6.8$, was used as solvent in the casting method. 200 $\mathrm{mg}$ of ATN and $200 \mathrm{mg}$ of HCZ were incorporated in mixtures containing different ratios and combinations of polymers and plasticizer. The matrices were prepared by pouring $10 \mathrm{ml}$ of the homogeneous solutions on the PVA-aluminium foil backing membrane. Then, these buccal patches were dried at $42{ }^{\circ} \mathrm{C}$ in an incubator (Yorco International Pvt. Ltd., India). After $24 \mathrm{~h}$, the dried patches were removed from the petri dishes and kept in desiccators until use [4]. The patches were cut into $2 \times 2 \mathrm{~cm}^{2}$ and $1 \times 1 \mathrm{~cm}^{2}$ for evaluation of the patches. Each patch size of $2 \times 2 \mathrm{~cm}^{2}$ contains 3.12 mg ATN and $3.12 \mathrm{mg} \mathrm{HCZ}$ respectively [4].

Table 1: Composition of different buccal patches

\begin{tabular}{|c|c|c|c|c|c|c|c|c|c|c|c|c|}
\hline \multirow[t]{2}{*}{ Ingredients } & \multicolumn{3}{|l|}{ FA } & \multicolumn{3}{|l|}{ FB } & \multicolumn{3}{|l|}{ FC } & \multicolumn{3}{|l|}{ FD } \\
\hline & 1 & 2 & 3 & 1 & 2 & 3 & 1 & 2 & 3 & 1 & 2 & 3 \\
\hline $\mathrm{HCZ}$ (mg) & 200 & 200 & 200 & 200 & 200 & 200 & 200 & 200 & 200 & 200 & 200 & 200 \\
\hline $\operatorname{ATN}(\mathrm{mg})$ & 200 & 200 & 200 & 200 & 200 & 200 & 200 & 200 & 200 & 200 & 200 & 200 \\
\hline $\mathrm{SA}(\mathrm{mg})$ & 300 & 200 & 100 & - & - & - & - & - & - & - & - & - \\
\hline HPMC (mg) & 200 & 300 & 400 & - & - & - & 200 & 300 & 400 & 300 & 200 & 100 \\
\hline Carbopol 934 (mg) & - & - & - & 300 & 200 & 100 & 300 & 200 & 100 & - & - & - \\
\hline $\mathrm{NaCMC}(\mathrm{mg})$ & - & - & - & 200 & 300 & 400 & - & - & - & 200 & 300 & 400 \\
\hline Glycerine (\%) & 15 & 15 & 15 & 15 & 15 & 15 & 15 & 15 & 15 & 15 & 15 & 15 \\
\hline Distilled Water (ml) & 40 & 40 & 40 & 40 & 40 & 40 & 40 & 40 & 40 & 40 & 40 & 40 \\
\hline
\end{tabular}

F-formulation, HCZ-hydrochlorothiazide, ATN-atenolol, SA-sodium alginate, HPMC-hydroxymethyl cellulose, NaCMC-sodium carboxymethyl cellulose

\section{Evaluation of buccal patches \\ Uniformity of weight}

Ten patches of $1 \mathrm{~cm}^{2}$ were weighed individually and average of those patches measured [5].

\section{Patch thickness}

The thickness of each patch was measured using screw gauge at five different positions of the patch and the average was calculated.

\section{Folding endurance}

Folding endurance of the patches was determined by repeatedly folding one patch at the same place till it broke or folded up to 300 times manually, which was considered satisfactory to reveal good patch properties. The number of times of patch could be folded at the same place without breaking gave the value of the folding endurance. This test was done on five patches [6].

\section{Surface $\mathrm{pH}$ of the buccal patches}

The surface $\mathrm{pH}$ of the patches was determined to investigate the possibility of any side effects due to change in $\mathrm{pH}$ in vivo, since an acidic or alkaline $\mathrm{pH}$ may cause irritation to the buccal mucosa. The patch to be tested was placed in petri dish and was moistened with $0.5 \mathrm{ml}$ of distilled water and kept for $30 \mathrm{~s}$. The $\mathrm{pH}$ was noted after bringing an electrode of PH meter in contact with the surface of the formulation and allowing equilibrating for $1 \mathrm{~min}[6]$.

\section{Measurement of bioadhesive strength}

Bioadhesive strength refers to mechanical strength of the system. Bioadhesive strength test was conducted to check the residence time of the patch at the site of application. The tensile strength required to detach the bioadhesive patch from the mucosal surface was reported as a parameter of the bioadhesive performance. In the present work, a specially designed or fabricated assembly based on published literature was used. Porcine cheek pouch was used as a model surface for bioadhesion testing. After the cheek pouch was excised and trimmed evenly, it was then washed in simulated salivary fluid, and then used immediately [7].

\section{Swelling index}

The swelling index procedure was used to determine the general swelling characteristics of patch. A drug-loaded patch of $1 \times 1 \mathrm{~cm}^{2}$ was weighed on a pre-weighed cover slip. It was kept in a petridish and $50 \mathrm{ml}$ of phosphate buffer, $\mathrm{pH} 6.8$ was added. After every five $\mathrm{min}$, the cover slip was removed and weighed up to $30 \mathrm{~min}$. The difference in the weights gives the weight increase due to absorption of water and swelling of patch. Area increase due to swelling: A drug loaded patch size of $1 \times 1 \mathrm{~cm}^{2}$ was cut and placed in a petridish. A graph paper was placed beneath the petridish, to measure the increase in the area. Fifty $\mathrm{ml}$ of phosphate buffer, $\mathrm{pH}$ 6.6, was poured into the petridish. An increase in the length and breadth of the patch was noted at five min intervals for $60 \mathrm{~min}$ and the area was calculated. [8] The swelling index was calculated using the following equation:

$$
\text { Swelling index }=\frac{W t-W 0}{W 0}
$$

Where, $W t$ is weight of the patch at time $t$ and $W O$ is weight of the patch at time zero.

\section{Drug content}

Prepared buccal patch was dissolved in $100 \mathrm{ml}$ of Phosphate buffer solution (PBS) of pH 6.8 using a magnetic stirrer for $12 \mathrm{~h}$ and then sonicated for $30 \mathrm{~min}$ designed to contain each $200 \mathrm{mg}$ of HCZ and ATN. After filtration to remove insoluble residue, $1 \mathrm{ml}$ of filtrate was diluted to $10 \mathrm{ml}$ with phosphate buffer $\mathrm{pH}$ 6.8. The absorbance was measured at $269.6 \mathrm{~nm}$ and $237.3 \mathrm{~nm}$ respectively for HCZ and ATN using UV spectrophotometer. The percentage drug content of all formulations was found to be in the range of $97-99 \%[7,8]$.

\section{Measurement of tensile strength (TS) and Percentage elongation (E/B)}

The mechanical property was evaluated using instron universal testing instrument (Model 1121, instron Ltd., japan, NITK, Surathkal, India) with a $5 \mathrm{~kg}$ load cell. Film strips in special dimension and free from air bubbles or physical imperfections were held between two clamps positioned at a distance of $3 \mathrm{~cm}$. During the measurement, the strips were pulled by the top clamp at a rate of $100 \mathrm{~mm} / \mathrm{min}$, and then the force and elongation were measured when the film broke. Results from the film samples, which broke at and not between the clamps, were not included in the calculations. Measurements were run in triplicate for each film. Two mechanical properties, namely, TS and \% E/B were computed for the evaluation of the film. TS is the maximum stress applied to a point at which the film specimen breaks and was computed from the applied load at rupture as a mean of three measurements and cross sectional area of fractured film as described from the following equation.

Tensile strength $=\frac{\text { Force at break }}{\text { Initial cross section area of the sample (mm2) }}$ 


$$
\text { Percentage elongation }=\frac{\text { Increase in length }}{\text { Original length }} \times 100
$$

\section{In vitro release study}

A patch of $2 \times 2 \mathrm{~cm}^{2}$ size was cut and attached to a glass slide with a few drops of phosphate buffer ( $\mathrm{pH}$ 6.8). This slide was kept at an angle of $45^{\circ} \mathrm{C}$ in a $250 \mathrm{ml}$ beaker containing $100 \mathrm{ml}$ of phosphate buffer $\mathrm{(pH}$ 6.8) solution. The beaker was kept in circulating water bath in which the temperature was maintained at $37^{\circ} \mathrm{C}$. A non-agitated system was selected to eliminate any effect of turbulence on the release rate. Samples were withdrawn periodically after removing the slide from the beaker. The solution was stirred with a glass rod and $5 \mathrm{ml}$ of sample was withdrawn using a graduated pipette, whose tip was attached to a tube with glass wool (as a filter). The slide was quickly reintroduced into the beaker. $5 \mathrm{ml}$ of the buffer was replaced immediately and the beaker was kept covered with a petridish to prevent evaporation of the fluid. The samples were taken after every $10 \mathrm{~min}$ upto $90 \mathrm{~min}$ and analysed for drug contents.

The withdrawn samples were analyzed spectrophotometrically at $235.5 \mathrm{~nm}$ and $264.5 \mathrm{~nm}$ for

With suitable dilution, samples were measured for absorbance using UV-Visible spectrophotometer $235.5 \mathrm{~nm}$ and $264.5 \mathrm{~nm}$ for HCZ and ATN respectively by using Shimadzu double beam ultra violet-visible spectrophotometer (UV-1700, Shimadzu Corporation, Tokyo, Japan). The release studies were conducted for three times and average was determined for HCZ and ATN $[9,10]$.

\section{In vitro diffusion study}

In vitro diffusion study was performed by using modified franz diffusion cell across cellophane membrane. Phosphate buffer solution (PBS) of $\mathrm{pH} 6.8$ was used as medium for diffusion study. Patches of dimension $1 \times 1 \mathrm{~cm}^{2}$ were placed on the membrane, which was placed between donor and receptor compartment of Franz diffusion cell. Cellophane membrane was brought in contact with PBS of pH 6.8 filled in receptor compartment. Temperature was maintained at $37{ }^{\circ} \mathrm{C}$ with stirring at $50 \mathrm{rpm}$ using magnetic bead stirrer. $1 \mathrm{ml}$ of sample was withdrawn from receptor compartment at pre-determined interval and was replaced with fresh PBS of $\mathrm{pH}$ 6.8. With suitable dilution, samples were measured for absorbance using UV-Visible spectrophotometer 235.5 and $264.5 \mathrm{~nm}$ respectively for HCZ and ATN $[11,12]$.

\section{Ex vivo permeability study}

The extent and rate of mucosal permeation of HCZ and ATN through the porcine buccal mucosa were carried out using Franz diffusion cell. The effective diffusion area was $1.8 \mathrm{~cm}^{2}$. The receptor compartment $(40 \mathrm{ml})$ was filled with PBS, $\mathrm{pH} 6.8$, and its temperature was maintained at $37 \pm 0.5{ }^{\circ} \mathrm{C} .50 \mathrm{rpm}$ stirring speed was applied using a magnetic stirrer to simulate buccal cavity environment. The patch of $1 \times 1 \mathrm{~cm}^{2}$ was applied under occlusion on the buccal mucosal surface of the goat fitted between the donor and receptor compartments of the diffusion cell. $5 \mathrm{ml}$ of the sample from receptor medium was withdrawn at regular intervals and replaced immediately with an equal volume of PBS, $\mathrm{pH}$ 6.8. The amount of HCZ and ATN released into the receptor medium was quantified by using UV-Visible Spectrophotometer at 235.5 and $264.5 \mathrm{~nm}$ respectively against a blank $[13,14]$.

\section{Kinetic analysis}

To analyse the mechanism of the drug release rate kinetics of the dosage form, the data obtained were fitted into \% cumulative drug release (CDR) vs time for Zero order kinetics, Log \% CDR remaining vs time for First order kinetics, \% CDR vs Square root of time for Higuchi model and Log \% CDR vs Logt for Korsmeyer model were plotted to obtain $\mathrm{R}^{2}$ values [15].

\section{Stability study}

Stability testing of drug products begins as a part of drug discovery and ends with the demise of the compound or commercial product. Food and Drug Administration (FDA) and International conference of Harmonization (ICH) specifies the guidelines for stability testing of new drug products, as a technical requirement for the registration of pharmaceuticals for human use. The ICH Tripartite Guidelines have established that long term stability testing should be done at $25^{\circ} \mathrm{C} / 60 \%$ (Relative Humidity) RH; stress testing should be done at $40{ }^{\circ} \mathrm{C} / 75 \% \mathrm{RH}$ for $6 \mathrm{mo}$. If significant change occurs at these stress conditions, then the formulation should be tested at an intermediate condition i.e. $30{ }^{\circ} \mathrm{C} / 75 \%$ RH. In the present study stability studies were carried out at two different temperatures i.e., refrigeration temperature $\left(2 \pm 8^{\circ} \mathrm{C}\right)$ and room temperature $\left(25 \pm 30^{\circ} \mathrm{C}\right)[14,15]$.

\section{RESULTS AND DISCUSSION}

\section{Compatibility studies}

IR studies were carried out for pure drugs and excipients which were used in formulations to determine the interaction between drug and polymers The IR spectra are given in the fig. 1 a,b,c and d. The spectral values for the drugs were compared with reference standard sample spectra. The IR spectrum of the HCZ showed the characteristic peaks at $3362.04 \mathrm{~cm}^{-1}$ (NH stretching group), 1604.83 $\mathrm{cm}^{-1}$ (C-C stretching group), $1244.13 \mathrm{~cm}^{-1}$ ( $\mathrm{SO}_{2}$ stretching group) and $2690 \mathrm{~cm}^{-1}\left(-\mathrm{CH}_{2}\right.$ group). The IR spectrum of the ATN showed the characteristic peaks at $1650 \mathrm{~cm}^{-1}\left(\mathrm{O}=\mathrm{C}-\mathrm{NH}_{2}\right.$ group), $2880-2900 \mathrm{~cm}^{-1}$ (CH group), 2916-2936 $\mathrm{cm}^{-1}$ ( $\mathrm{CH}_{2}$ group), $2850 \mathrm{~cm}^{-1}$ ( $\mathrm{CH}_{3}$ group), $1640-1610 \mathrm{~cm}^{-1}$ ( $\mathrm{C}=\mathrm{C}$ group), $3200-3550 \mathrm{~cm}^{-1}$ (-OH group). The spectra of formulations showed presence of peaks in the region of characteristic peaks of drugs confirmed the absence of interaction between the drugs and excipients used in the formulation.

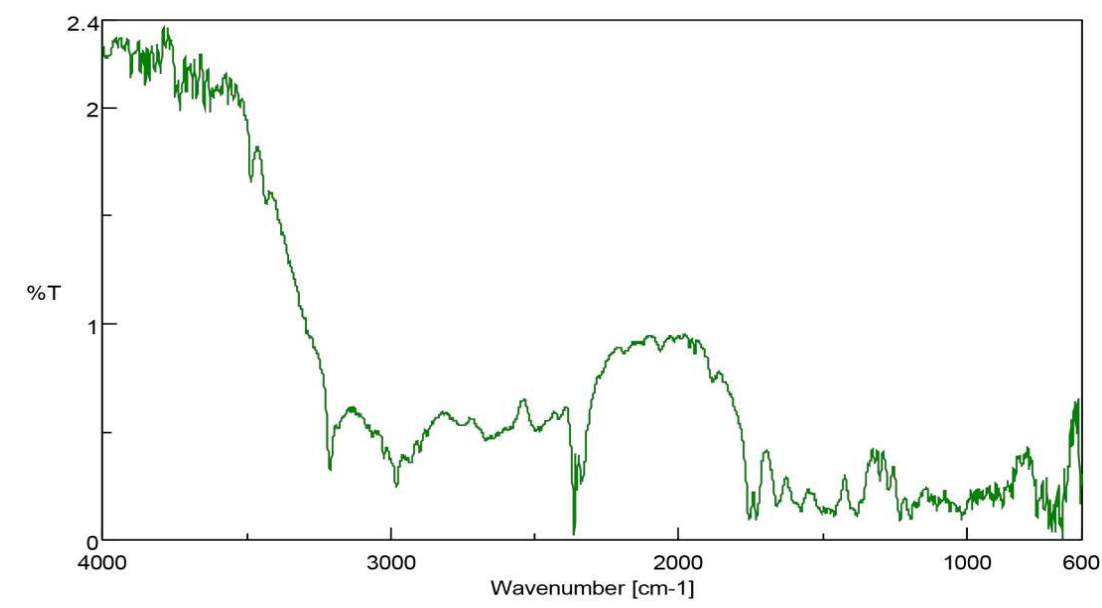

Fig. 1a: FTIR spectra of pure HCZ 


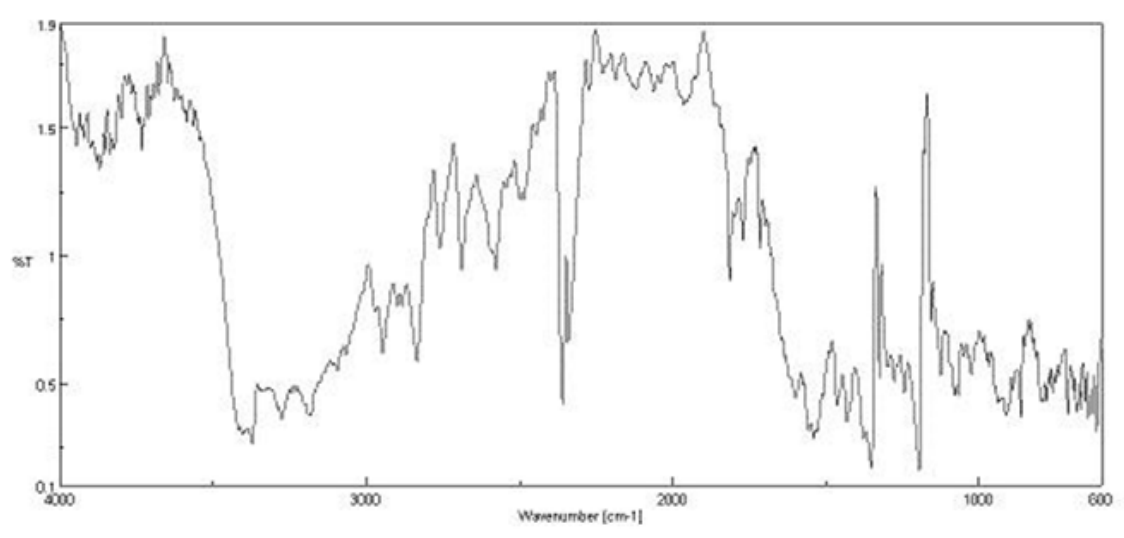

Fig. 1b: FTIR spectra of pure ATN

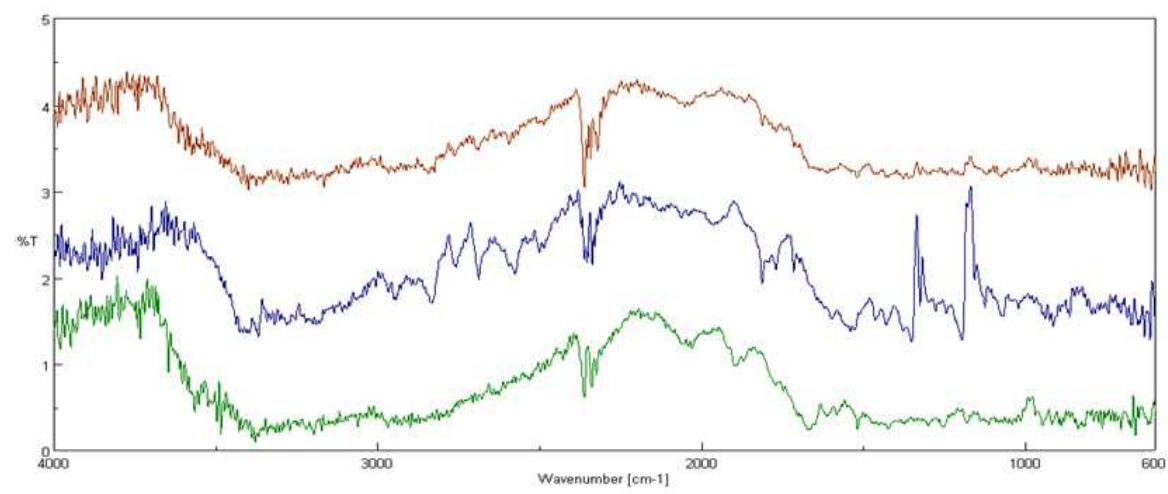

Fig. 1c: FTIR spectra of of HCZ, ATN and F3 formulation

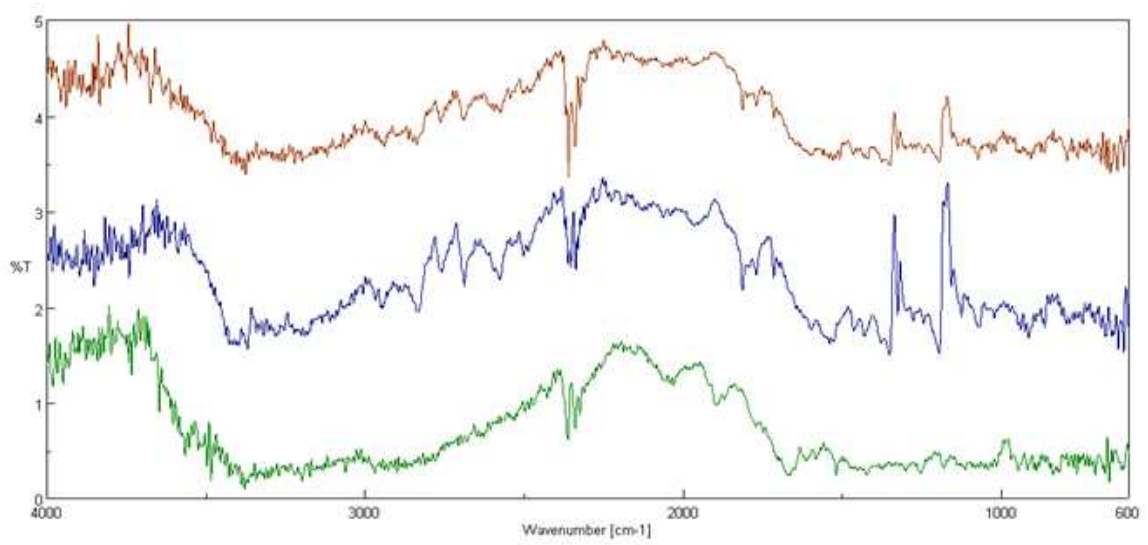

Fig. 1d: FTIR spectra of of HCZ, ATN and F4 formulation

\section{Physico-chemical evaluation of prepared buccal patches}

All formulated patches were found to be smooth in texture and transparent. The individual weight of 3 formulations of each type were determined and the average weight was calculated. It was observed that the weight of the patches in each formulation was found to be uniform. The thickness of the patches of each formulation was determined using micrometer screw gauge. It was observed that the thickness of all patch samples were found to be uniform in each formulation. The patches with increased polymer content showed a marginal increase in thickness. The folding endurance was determined as per the procedure mentioned in the methodology. It was found that all the formulations showed good folding endurance greater than 300 . The surface $\mathrm{pH}$ of the patches was also determined and observed that the surface $\mathrm{pH}$ of each patch was found between 6.15 to 6.66 and which means that they may have less potential to irritate the buccal mucosa, as a result patches will be compatible to mucosa. The percentage drug content of all formulations was found to be in the range of 97-99 $\%$. Among the formulations prepared, it was observed that FC takes longer time to disintegrate since it contains Carbopol 934 and HPMC which is highly viscous at increased concentration [16, 17]. The results for all formulations and the average of 3 determinations are given in table 2 . 
Table 2: Results of physic-chemical parameters

\begin{tabular}{|c|c|c|c|c|c|c|}
\hline \multicolumn{2}{|c|}{ Formulation code } & \multirow{2}{*}{$\begin{array}{l}\text { Weight (mg)* } \\
39 \pm 0.04\end{array}$} & \multirow{2}{*}{$\begin{array}{l}\text { Thickness (mm)* } \\
0.54 \pm 0.02\end{array}$} & \multirow{2}{*}{$\begin{array}{l}\text { Surface pH } \\
6.3\end{array}$} & \multirow{2}{*}{$\begin{array}{l}\text { Folding endurance } \\
326\end{array}$} & \multirow{2}{*}{$\begin{array}{l}\text { Drug content (\%) } \\
30 \pm 0.10\end{array}$} \\
\hline FA & 1 & & & & & \\
\hline & 2 & $38 \pm 0.03$ & $0.56 \pm 0.03$ & 6.2 & 314 & $35 \pm 0.12$ \\
\hline & 3 & $40 \pm 0.04$ & $0.57 \pm 0.02$ & 6.3 & 339 & $40 \pm 0.13$ \\
\hline \multirow[t]{3}{*}{ FB } & 1 & $45 \pm 0.02$ & $0.53 \pm 0.04$ & 6.1 & 348 & $40 \pm 0.04$ \\
\hline & 2 & $46 \pm 0.05$ & $0.53 \pm 0.02$ & 6.2 & 330 & $45 \pm 0.06$ \\
\hline & 3 & $48 \pm 0.06$ & $0.54 \pm 0.05$ & 6.3 & 335 & $60 \pm 0.06$ \\
\hline \multirow[t]{3}{*}{$\mathrm{FC}$} & 1 & $48 \pm 0.02$ & $0.58 \pm 0.02$ & 6.4 & 331 & $45 \pm 0.07$ \\
\hline & 2 & $46 \pm 0.04$ & $0.56 \pm 0.03$ & 6.6 & 333 & $50 \pm 0.07$ \\
\hline & 3 & $47 \pm 0.06$ & $0.55 \pm 0.01$ & 6.3 & 350 & $60 \pm 0.07$ \\
\hline \multirow[t]{3}{*}{ FD } & 1 & $49 \pm 0.03$ & $0.54 \pm 0.03$ & 6.2 & 310 & $30 \pm 0.08$ \\
\hline & 2 & $48 \pm 0.04$ & $0.51 \pm 0.04$ & 6.1 & 315 & $35 \pm 0.08$ \\
\hline & 3 & $44 \pm 0.02$ & $0.59 \pm 0.02$ & 6.3 & 325 & $45 \pm 0.12$ \\
\hline
\end{tabular}

${ }^{*} \mathrm{n}=3$, SD-standard deviation, F-formulation

\section{Measurement of swelling index}

The hydration and swelling behavior of the polymer was reported to be crucial for its bio adhesive character because the former is necessary to initiate intimate contact of the patch with the mucosal surface. The adhesion increases with the degree of hydration until a point where over hydration leads to an abrupt drop in adhesive strength due to disentanglement at the polymer tissue interface. The rate and the extent of patch hydration and swelling also affects the patch adhesion and consequently the drug release from the patch. Studies have shown that excessive hydration can lead to weakening of the adhesive bond due to dilution of functional groups responsible for the adhesive interaction between the bio adhesive patch and mucosa. It was found that the increase in swelling of patches formulated with Carbopol 934 and HPMC was more compared to other formulations. This result may be attributed to complete penetration of solvent and high viscosity of the carbopol 934. The obtained results showed that the swelling front erosion was comparably slower in formulation batch with Carbopol 934 and HPMC due to their marked viscosity properties. Hence the formulation provides sustained release of drug. The poor solubility of SA limits the swelling of the patch [17] (fig. 2).

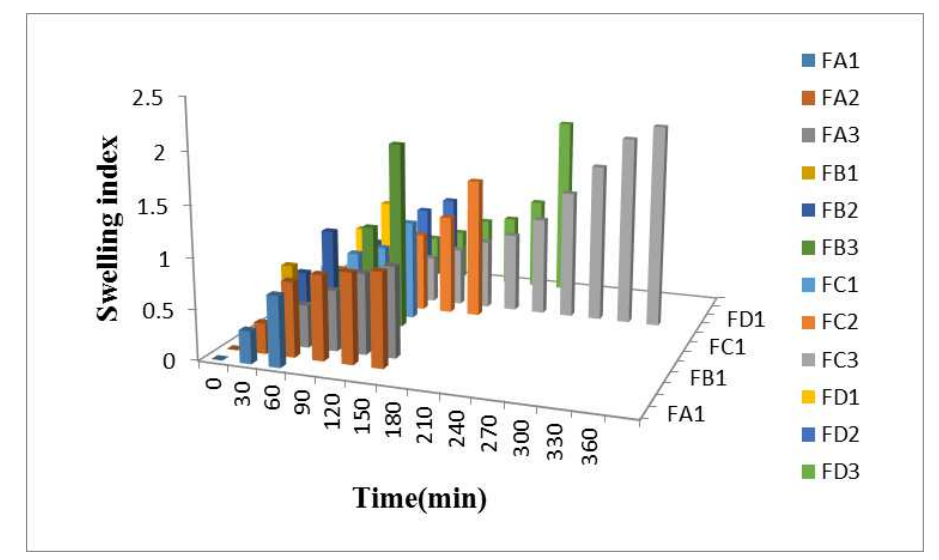

Fig. 2: Swelling index, (All the values were calculated as mean \pm standard deviation; $\mathbf{n = 3}$ )

\section{Bioadhesive strength}

In vitro mucoadhesive strength was determined on the modified balance to measure the force of adhesion (N) required to detach the tablet. From the overall study it was concluded that the mucoadhesive strength of polymers depend on their structure and other physicochemical properties. As the mucoadhesive polymer mixture concentration increased, the mucoadhesive strength and force of adhesion increased. Very strong mucoadhesion could damage the epithelial lining of the buccal mucosa. Among all the formulations FC (Carbopol 934 and HPMC) has higher mucoadhesive strength and higher the force required to detach from mucosa. Therefore FC patches can be considered satisfactory for maintaining them in the oral cavity for 8h. Among FA and FD maximum bioadhesion was observed for SA patches $[18,19]$.

\section{Tensile strength (TS) and percentage elongation (E/B)}

The TS gives an indication of the strength and elasticity of the patch. A weak and soft polymer is characterized by a low TS and E/B, a hard and brittle polymer shows a moderate TS and low E/B, a soft and tough polymer is characterized by a moderate TS and high E/B whereas a hard and tough polymer shows a high TS and E/B. The results showed that, among the formulation FA to FD, TS and E/B increased with the increase in the percentage of polymers. Formulations FD (with CP and NaCMC) and FC (Carbopol 934 and HPMC) showed high Tensile strength and \% E/B than FA (with SA and HPMC) and FB (HPMC and NaCMC) which indicated that FC and FD gives good tensile strength to buccal patches which are tough and strong enough for use compared to FA and FB. In case of formulations FA and FD, FA (SA and HPMC) at higher concentration showed TS and $\mathrm{E} / \mathrm{B}$ greater compared to formulation FD (CP and NaCMC), indicated that the inclusion of $\mathrm{CP}$ decreased the tensile strength. This indicates that the presence of water soluble polymers tend to make the polymer softer and less tough and therefore poor TS and E/B [19] (table 3).

\section{In vitro release studies}

Amongst the formulations FC2 containing carbopol 934 and HPMC (2:3) released the drug in sustained form i. e, $95 \%$ at $8 \mathrm{~h}$ since carbopol 934 at higher concentration created a high viscosity gel barrier for drug diffusion. Increased concentrations of NaCMC resulted faster drug release. This result was attributed to water soluble polymer NaCMC, results in increased wettability and penetration of water into the patch matrices and hence increased diffusion of the drug [20] (fig. 3 and 4). 
Table 3: Tensile strength and \% elongation of different formulations

\begin{tabular}{lll}
\hline Formulation code & Tensile strength $\mathbf{~} \mathbf{K g} / \mathbf{m m}^{\mathbf{2}} \mathbf{)}$ & \% Elongation \\
\hline FA 1 & $0.89 \pm 0.01$ & $37.5 \pm 0.03$ \\
2 & $0.79 \pm 0.02$ & $39.1 \pm 0.04$ \\
3 & $0.85 \pm 0.03$ & $55.0 \pm 0.05$ \\
FB 1 & $0.65 \pm 0.02$ & $19.0 \pm 0.02$ \\
2 & $0.60 \pm 0.02$ & $20.0 \pm 0.01$ \\
3 & $0.62 \pm 0.01$ & $25.7 \pm 0.03$ \\
FC 1 & $1.15 \pm 0.03$ & $90.1 \pm 0.01$ \\
2 & $1.17 \pm 0.03$ & $89.7 \pm 0.04$ \\
3 & $1.19 \pm 0.02$ & $85.2 \pm 0.05$ \\
FD 1 & $0.95 \pm 0.01$ & $57.0 \pm 0.01$ \\
2 & $0.98 \pm 0.01$ & $59.0 \pm 0.01$ \\
3 & $0.94 \pm 0.02$ & $60.0 \pm 0.04$ \\
\hline
\end{tabular}

$\mathrm{n}=3$, SD-standard deviation, F-formulation, \%-percentage

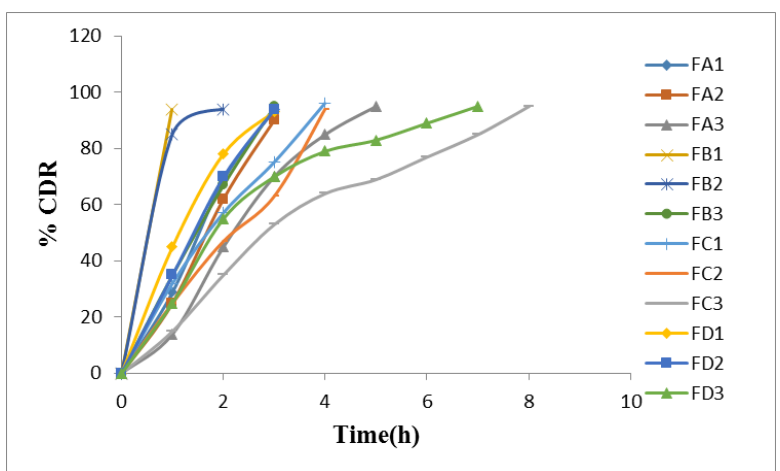

Fig. 3: In vitro drug release of HCZ, (Results are expressed as as mean \pm standard deviation; $n=3$ )

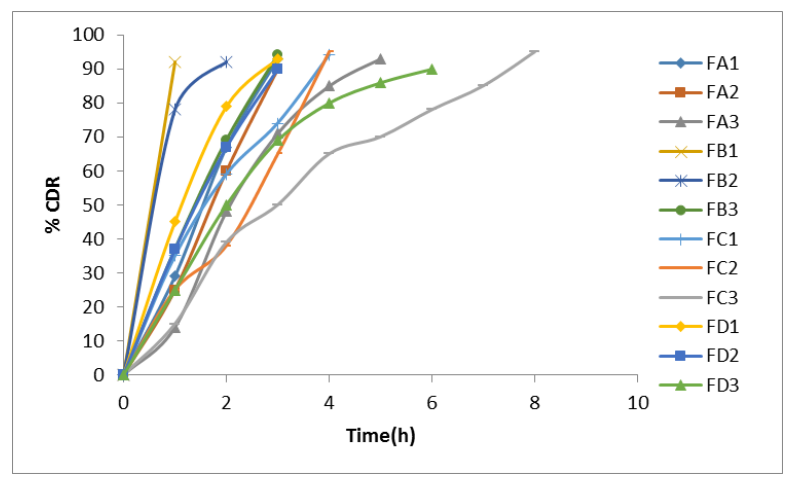

Fig. 4: In vitro drug release of ATN, (Results are expressed as as mean \pm standard deviation; $n=3$ )

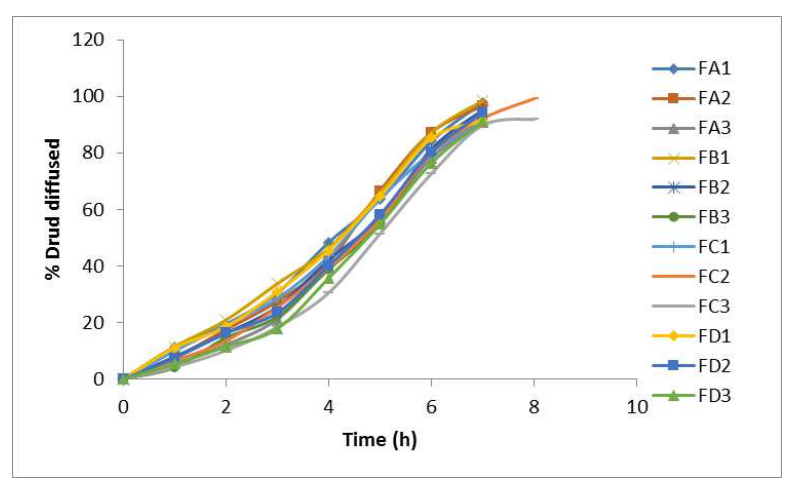

Fig. 5: In vitro diffusion of HCZ, (Results are expressed as as mean \pm standard deviation; $\mathrm{n}=3$ )

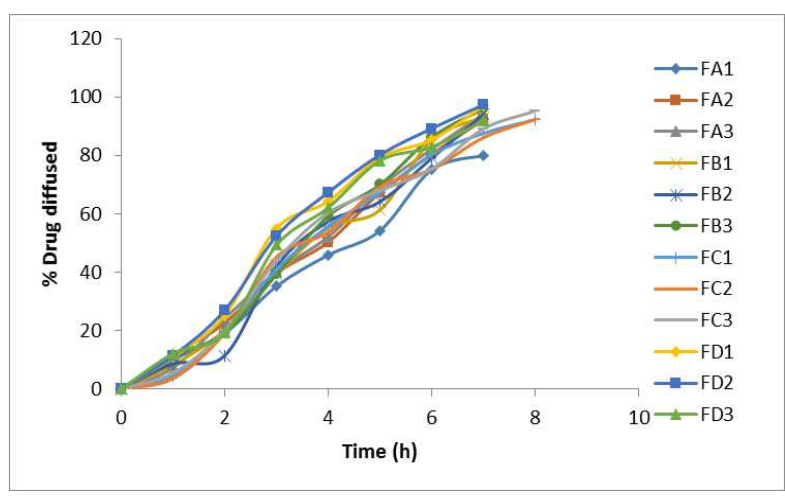

Fig. 6: In vitro diffusion of ATN, (Results are expressed as as mean \pm standard deviation; $\mathbf{n}=3$ )

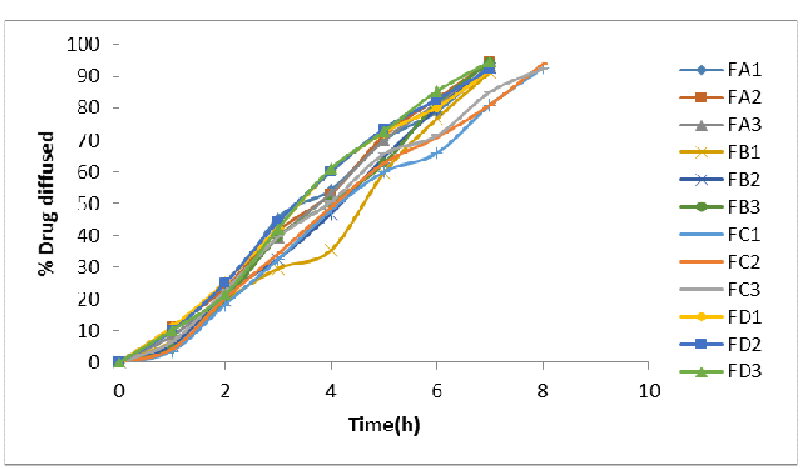

Fig. 7: In vitro drug permeation of HCZ, (Results are expressed as as mean \pm standard deviation; $n=3$ )

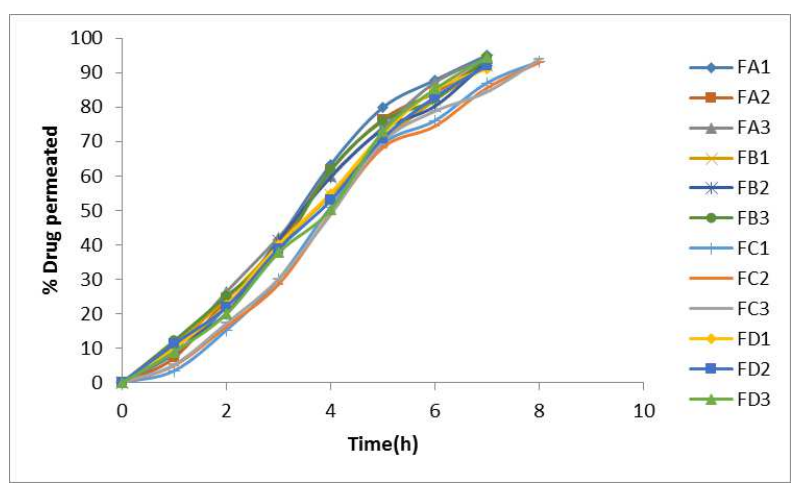

Fig. 8: In vitro drug permeation of ATN, (Results are expressed as as mean \pm standard deviation; $n=3$ ) 


\section{In vitro diffusion studies}

It was found that in FC 2 formulation containing carbopol 934 and HPMC (2:3) was found to be comparable with the in vitro diffusion study data and hence indicated a good diffusion coefficient which is essential for all such drugs which are formulated for the purpose of sustained formulations. Hence FC 2 was found to be better amongst the formulation [21]. This may be attributed due to increasing concentration of polymers [21] (fig. 5 and 6).

\section{Ex vivo permeation studies}

It was observed that, as the polymer content increased, the \% drug permeation decreased. The formulation containing Carbopol 934 and HPMC showed better permeation compared to all other formulations. It was found that the results obtained in ex vivo study indicated that drug has the better ability to cross the buccal barrier at a faster rate and hence the delivery system has the potential of overcoming the drawbacks associated with presently available tablet formulations in the market (fig. 7 and 8).

\section{Kinetic analysis}

In order to determine the release mechanism that provides the best description to the pattern of drug release, the in vitro release data were fitted to Zero order, First order, and Higuchi model. The release data were also kinetically analysed using the KorsmeyerPeppas model. The data were processed for regression analysis using MS-EXCEL statistical function [22] (table 4).

Table 4: Kinetic release of different formulations

\begin{tabular}{|c|c|c|c|c|c|}
\hline Formulation code & Zero order $\mathbf{R}^{2}$ & First order $\mathbf{R}^{2}$ & Higuchi model R ${ }^{2}$ & Korsmeyer peppas model & $\mathbf{N ~ R}^{2}$ \\
\hline FA 1 & 0.9736 & 0.9963 & 0.7122 & 0.9656 & 0.4535 \\
\hline 2 & 0.9798 & 0.9950 & 0.6968 & 0.9776 & 0.4578 \\
\hline 3 & 0.9798 & 0.9866 & 0.6977 & 0.9780 & 0.4598 \\
\hline FB 1 & 0.9657 & 0.9933 & 0.6736 & 0.9638 & 0.4637 \\
\hline 2 & 0.9783 & 0.9960 & 0.6923 & 0.9778 & 0.4789 \\
\hline 3 & 0.9702 & 0.9897 & 0.6886 & 0.9734 & 0.4739 \\
\hline FC 1 & 0.9766 & 0.9933 & 0.7210 & 0.9938 & 0.4761 \\
\hline 2 & 0.9826 & 0.9976 & 0.6825 & 0.9866 & 0.4839 \\
\hline 3 & 0.9839 & 0.9878 & 0.6999 & 0.9880 & 0.4817 \\
\hline FD 1 & 0.9656 & 0.9945 & 0.6856 & 0.9333 & 0.4839 \\
\hline 2 & 0.9742 & 0.9913 & 0.6890 & 0.9413 & 0.4981 \\
\hline 3 & 0.9668 & 0.9955 & 0.6843 & 0.9766 & 0.4937 \\
\hline
\end{tabular}

F-formulation

By using Korsmeyer-Peppas model, if $n=0.45$ it is Case- 1 or Fickian diffusion, $0.45<n>0.89$ is for anomalous behavior or nonfickian transport, $n=0.89$ for Case 11 transport, and $n>0.89$ for Super Case 11 transport. Fickian release usually occurs by molecular diffusion of the drug due to a chemical potent gradient. Case 11 relaxation release is the drug transport mechanism associated with stresses and state transition in hydrophilic glassy polymers, which swell in water or biological fluids. This term also includes polymer disentanglement and erosion. In the present investigation, the release from the polymers followed anomalous behavior or nonfickian transport. As a result the combination of diffusion and erosion was the mechanism followed by the formulations as the ' $n$ ' values ranged from 0.4535 to 0.4937 as per Korsmeyer-Peppas model, which in turn justified suitability of polymers for the preparation of buccal patches [22]

\section{Stability studies}

Stability testing was carried out for all the formulations for a period of eight weeks. All the formulations were evaluated with respect to physical appearance, drug content, surface $\mathrm{pH}$, swelling index and in vitro drug release. The results of stability studies of HCZ and ATN buccal patches showed no significant change with respect to physical appearance, drug content, surface $\mathrm{pH}$, swelling index and in vitro drug release at the end of eight weeks when stored in refrigeration temperature $\left(2 \pm 8{ }^{\circ} \mathrm{C}\right)$ and room temperature $(25 \pm 30$ ${ }^{\circ} \mathrm{C}$ ). Ageing did not alter the drug release profiles of any of the patches significantly till the end of the storage period. Buccal patches were found to be physically and chemically stable.

\section{CONCLUSION}

Sustained release patch may open a new horizon in buccal drug delivery system. In the present investigation, an attempt was made to improve the systemic bioavailability, avoidance of pre-systemic elimination within the GI tract and optimize therapeutic efficacy of the selected drugs by designing carefully mucoadhesive buccal patches for control release of HCZ and ATN. HCZ and ATN are available in combination in conventional tablet form in a market and are effective in the combination for the treatment of hypertension. Hence this drug combination will benefit from formulation into mucoadhesive buccal patches by avoiding first pass metabolism and therefore improvement in bioavailability and also improves patient compliance by reducing dosing frequency.

\section{ACKNOWLEDGEMENT}

The authors wish to acknowledge the Shree Devi college of Pharmacy, Mangalore, India for providing necessary facilities and financial support to carry out this project. The authors are also thankful to Bhagwant University for accepting the research project. The authors are thankful to Department of Pharmaceutics, NGSM Institute of Pharmaceutical Sciences, Nitte University, Mangalore for the logistic support and guidance.

\section{AUTHORS CONTRIBUTIONS}

All authors have contributed equally

\section{CONFLICT OF INTERESTS}

Declared none

\section{REFERENCES}

1. Hoogstrate AJ, Verhoef JC, Tuk B, Pijpers A, Van Leengoed LA, Verheijden $\mathrm{JH}$, et al. In vivo buccal delivery of fluorescein isothiocyanate-dextran 4400 with glycodeoxycholate as an absorption enhancer in pigs. J Pharm Sci 1996;85:457-60.

2. Patel VM, Prajapati BG, Patel MM. Design and characterization of chitosan containing mucoadhesive buccal patches of propranolol hydrochloride. Acta Pharm 2007;57:61-72.

3. Vashmi Vishnu Y, Chandrasekhar K, Ramesh G, Madhusudan Rao Y. Development of mucoadhesive patches for buccal administration of carvedilol. Curr Drug Delivery 2007;4:27-39.

4. Khairnar A, Jain P, Baviskar D, Jain D. Development of mucoadhesive buccal patch containing aceclofenac: in vitro evaluation. Int J Pharm Sci 2009;1:91-5.

5. Hao J, Heng PW. Buccal delivery systems. Drug Dev Ind Pharm 2003;29:821-32.

6. Pankil AG, Patel MR, Patel KR, Patel NM. A review article on mucoadhesive buccal drug delivery system. Int J Pharm Res Dev 2011;3:121-9. 
7. Birudaraj R, Berner B, Shen S, Li X. Buccal permeation of busipirone: mechanistic studies on transport pathways. J Pharm Sci 2005;94:70-8.

8. Harris D, Robinson JR. Bioadhesive polymers in peptide drug delivery. Biomaterials 1990;11:652-8.

9. Kumar TP, Desai KG, Kumar SG. Mechanism of buccal permeation enhancers. Indian J Pharm Edu 2002;36:147-51.

10. Gandhi SD, Pandya PR, Umbarkar R, Tambawala T, Shah MA. Mucoadhesive drug delivery system-an unusual maneuver for site specific drug delivery system. Int J Pharm Sci 2011;14:851-72.

11. Angela A, Federica B, Teresa C, Federica C, Beatrice V, Barbara L. Mucoadhesive chitosan/gelatin films for buccal delivery of propranolol hydrochloride. Carb Pol 2011;29:821-32.

12. Patel KV, Patel ND, Dodiya HD, Shelat PK. Buccal bioadhesive drug delivery system: an overview. Indian J Pharm Biol Res $2011 ; 2: 600-9$

13. Satyabrata B, Ellaiah P, Choudhury R, Murthy KVR, Bibhutibhusan P, Kumar MS. Design and evaluation of methotrexate buccal mucoadhesive patches. Int J Pharm Biomed Sci 2010;1:31-6.

14. Patil BS, Tate SS, Kulkarni U, Hariprasanna RC, Wadageri GV. Development and in vitro evaluation of mucoadhesive buccal tablets of tizanidine hydrochloride using natural polymer Xanthan gum. Int J Pharm Sci Rev Res 2011;8:140-6.
15. Elk YR, Friedman M, Stabholz A, Soskolne AW, Sela MN, Golub L. Sustained release device containing minocycline for local treatment of periodontal disease. J Controlled Release 1998; 7:231-6.

16. Ishida M, Nambu N, Nagai T. Mucosal dosage form of lidocaine for toothache using hydroxypropyl cellulose and carbopol. Chem Pharm Bull 1982;30:980-4.

17. Sadeq ZA, Rajab NA. Studying the effect of different varaiables on the formulation of mucoadhesive buccal patches of captopril. Int J Appl Pharm 2017;9:16-21.

18. Singh RK, Shah JN, Mehta TA. Mucoadhesive bilayer buccal patches of verapamil hydrochloride: formulation, development and characterization. Int J Pharm Pharm Sci 2014;6:234-2.

19. Das R. Effective mucoadhesive buccal patches: wound healing activity of curcumin and centella asiatica extract compared to rhEGF. Int J Pharm Pharm Sci 2011;3:97-100.

20. Rasool BKA, Khan SA. In vitro evaluation of miconazole buccal films. Int J Appl Pharm 2010;2:23-6.

21. Elbary AA, Makky AMA, Tadros MB, Ahmed AA. Development and in vitro evaluation of mucoadhesive bilayer buccal tablets of carvedilol. Int J Pharm Pharm Sci 2015;7:172-6.

22. Ritger PL, Peppa NA. A simple equation of solute release 1. Fickian and anamolous from swellable devices. J Controlled Release 1987;5:23-36. 